\title{
Improving Well-Being and Survival in the 6-OHDA Lesion Model of Parkinson's Disease in Mice: Step- By-Step Protocol
}

\section{Adriane Guillaumin}

Uppsala Universitet https://orcid.org/0000-0002-6488-3207

Bianca Vlcek

Uppsala Universitet

Åsa Wallén-Mackenzie ( $\square$ asa.mackenzie@ebc.uu.se )

Uppsala Universitet

\section{Research Article}

Keywords: Parkinson, mice, 6-OHDA, lesion, neurodegeneration, dopamine, survival, protocol

Posted Date: June 15th, 2021

DOl: https://doi.org/10.21203/rs.3.rs-603222/v1

License: (c) (i) This work is licensed under a Creative Commons Attribution 4.0 International License.

Read Full License 


\section{Abstract}

Background: Parkinson's disease (PD) is the most common neurodegenerative motor disorder and primarily affects movement control but also a range on non-motor functions. With unknown cause and lack of cure, much research is dedicated to find treatment. PD is characterized by progressive degeneration of midbrain dopamine neurons, primarily those of the substantia nigra pars compacta (SNc). Due to the complexity of the disease, animal models intended to represent symptoms similar to those observed in PD patients are instrumental to advance treatment prospects for PD. Among these, the 6-hydroxydopamine (6-OHDA) lesion model, in which dopaminergic neurons are chemically destroyed, is often favoured. However, while reproducing several features of clinical PD, including motor symptoms, mice exposed to 6-OHDA often suffer systemic dysfunction causing premature death. To avoid unnecessary spill of lives of laboratory mice and to increase the reliability of data obtained, there is a need for improved experimental protocols. Here we tested the effects of three parameters; lowered dose of toxin, careful post-operative care, and shortened interval between injection and sacrifice.

Results: A detailed 6-OHDA lesion protocol using lower dose of toxin than commonly seen in the literature alongside careful post-operative care and decreased time post-injection resulted in high survival rate. Successful degeneration of midbrain dopamine neurons was confirmed in the SNc using several markers, and nigrostriatal projections were lost in the lesioned hemisphere. Dopamine neurons of the ventral tegmental area (VTA) were substantially affected as well.

Conclusions: We demonstrate parameters that can be improved to increase well-being and survival of mice while preserving characteristic parkinsonian features in the 6-OHDA lesion model. A step-by-step protocol for implementation in any laboratory is provided.

\section{Background}

Parkinson's disease (PD) is the second most common progressive neurodegenerative disease with a prevalence of 1 to 2 per 1000 (1-3). PD patients are usually diagnosed around the age of 65 years, when motor symptoms begin to appear. Patients suffer from a progressive slowness in initiating movement, loss of postural reflexes and, with time, a rigidity of muscles. Some, but not all, PD patients also display resting tremor. A characteristic feature observed in the brain of PD patients is the presence of so called Lewy bodies, protein accumulation of misfolded a-synuclein (4-6). Another core feature of PD is the progressive and pathological degeneration of midbrain dopamine (DA) neurons, primarily DA neurons located in the substantia nigra pars compacta (SNc). With time, DA neurons of the ventral tegmental area (VTA), which project to limbic and cognitive neurocircuitry and which show higher resilience to PD, might also degenerate and additional functions are thereby affected.

SNc DA neurons project primarily to the dorsal striatum, forming the nigrostriatal pathway which is a critical modulator of motor control via the basal ganglia and associated structures (striatum, globus pallidus and the subthalamic nucleus (STN)). In healthy individuals, SNc DA neurons impact on the 
motor loop of the basal ganglia through the direct and indirect pathways. The direct pathway promotes movement while the indirect pathway suppresses movements. In PD, the absence of DA from the SNc causes the indirect pathway to become overactivated compared to the direct pathway, leading to the characteristic motor symptoms of PD. When motor symptoms appear and the affected individual becomes aware of them, $50-90 \%$ of SNc dopamine neurons have already been lost, as estimated by the presence of tyrosine hydroxylase, $\mathrm{TH}$, the rate-limiting enzyme of DA synthesis, and a commonly used marker for detection of these neurons (7).

Treatment strategies for PD patients mainly focus on replacing the loss of DA, either by pharmacological administration or cell replacement. For example, administration of DA agonists along with inhibitors of monoamine oxidase $B$ activity is often used in early stages of PD. Another treatment, the most common one, is the administration of levodopa (L-dopa), a precursor to DA. L-dopa is highly efficient in alleviating motor symptoms but has the unfortunate side-effect of inducing dyskinesia after prolonged use. Other potential treatments for PD includes various types of stem cell therapy which have in common the aim to replace degenerated dopamine neurons with newly generated dopamine neurons (8-10). Another type of PD treatment, which is not directed at replacing DA but rather at correcting aberrant basal ganglia activity, is deep brain stimulation (DBS). DBS consists of high-frequency electrical stimulation delivered via implanted electrodes positioned in relevant brain structures. The two most common brain targets for DBS electrodes in PD are the STN of the indirect motor pathway, and the internal segment of the globus pallidus (GPi) which is part of both the direct and indirect motor pathways (11). DBS has demonstrated a high success rate in alleviating motor symptoms of PD patients but some patients suffer from adverse effects (12-16).

While several treatment options exist for PD patients, none can cure the disease. Improved outcome in terms of alleviation of symptoms is highly desirable. Due to the complexity of the disorder, which affects the whole body as brain circuits become dysregulated, whole-animal experimental approaches are needed to reach relevant results. Several PD models using laboratory animals exist, most which claim to reproduce endophenotypes of PD rather than the whole complexity of the disease as displayed in humans. Animal PD models are based either on genetic mutations observed in patients (e.g. LRRK2, asynuclein), pharmacological interventions (reserpine, haloperidol (17)) or neurotoxins (N-methyl-4-phenyl1,2,3,6-tetrahydropyridine, MPTP; 6-hydroxydopamine, 6-OHDA). Currently existing animal PD models present one or several characteristics of the disease: Loss SNc DA neurons with the associated loss of $\mathrm{TH}$ immunoreactivity in the SNc and in nigrostriatal projections to the dorsal striatum; aggregation of misformed a-synuclein in Lewy bodies; aberrant activity of the STN; and/or impairment of motor behavior relevant to $\mathrm{PD}$, such as reduced locomotion, tremor, loss of balance and/or posture (18-20).

Neurotoxin-induced lesion models in rodents (rats and mice) are among the experimental animal models most used to induce PD-like symptoms. In particular, the 6-OHDA model mimics several symptoms of PD, including loss of spontaneous movement, DA cell death, and altered activity of the basal ganglia (2022). The 6-OHDA molecule resembles DA and is taken up by endogenous cytoplasmic membrane transport machinery of all catecholaminergic neurons. Inside the cell, two mechanisms of action are 
believed to cause cell death, the disturbance of the mitochondrial respiratory chain and the auto-oxidation process of the 6-OHDA molecule which leads to production of reactive oxygen species (23). 6-OHDA is usually injected unilaterally to allow one side of the brain to remain undisturbed by the deleterious effects of the toxin. Each animal thereby has one hemisphere with a normal midbrain DA system, an internal control, and one hemisphere with a lesioned DA system. Upon such unilateral injection of 6-OHDA, into either the SNc or the nigrostriatal pathway in the median forebrain bundle (MFB), the rodent displays a rotational behavior due to the induced loss of movement control exerted by the lesioned hemisphere. The efficiency of the model can thereby be ascertained efficiently by addressing rotational behavior, and its exacerbation by DA-releasing agents, such as the psyhcostimulant amphetamine, while the extent of DA cell degeneration requires post-mortem brain analysis, usually by addressing $\mathrm{TH}$ immunoreactivity in sections of the SNc and striatum.

When it comes to implementing the 6-OHDA lesion model in experimental rodents, ethical concerns are raised due to the frequent appearance of post-surgical death. The rodents experience difficulty in eating and maintaining body temperature as a result of the lesion. Ethical considerations commonly grade the procedure as of "severe difficulty". With our interest in generating an improved protocol for the 6-OHDA lesion model in laboratory mice, we searched the literature for standard doses used in rodents, common time post-lesioning until sacrifice, and procedures of post-operative care. To create conditions with high survival rate and well-being for 6-OHDA lesioned mice while maintaining efficient degeneration of the midbrain DA system, three lines of precautions were taken: 1) Reduced dose of the toxin; 2) Specific postoperative care; 3 ) Reducing post-lesioning time. The results show good survival rate of the mice despite adequate DA cell loss using lower dose of 6-OHDA than commonly used in the literature. Finally, there was equal cell loss after two weeks as after three weeks, suggesting that post-operative time prior to sacrifice can be reduced. The study contributes an experimental protocol in which animal safety has been taken into consideration, with findings suggesting that current protocols used can be improved using the results presented here.

\section{Results}

\section{Ipsilateral motor impairments and high survival rate upon low administration dose of 6-OHDA and post- operative care to prevent dehydration and hypothermia}

The concentration of 6-OHDA used in rodents as reported in the literature varies between 2.5 to $3.6 \mathrm{mg} / \mathrm{ml}$ free-base (24-26). Here we tested reducing this to $1.85 \mathrm{mg} / \mathrm{ml}$ free-base 6-OHDA. C57BL/6NTac mice were injected with 6-OHDA into the MFB of the right hemisphere and were subsequently divided into two groups depending on the duration of protocol, two or three weeks until sacrifice (Fig. 1). Immediately post-surgery, special considerations were taken to prevent dehydration and hypothermia, frequent sideeffects of the lesioning procedure as documented in the literature (24). Pre-heated saline was injected regularly which prevented hypothermia and sucrose was added to the cage, both as free liquid and on the food which enabled feeding throughout the procedure (Fig. 1A). Further, extra nutrition was supplied in the form of sunflower seed and gel-embedded nutrients. Weight loss was observed but rapidly stabilized 
after few days and increased for most mice around 7 days post-injection (Fig. 1B). A step-by-step protocol for the preparation of mice for surgery and the surgical procedure as well as post-operative care is provided (Additional file 1).

Despite the low dose of 6-OHDA used here, all mice displayed spontaneous ipsilateral rotations at various degrees, starting directly after the surgery and lasting until sacrifice. The spontaneous rotations thereby confirmed the successful lesion of the nigrostriatal pathway. Despite strong motor symptoms, a survival rate above $90 \%$ was obtained among the 10 mice injected with $6-$ OHDA, likely due to the daily postoperative care.

\section{6-OHDA injection induces a strong decrease of TH and additional dopaminergic markers in the lesioned hemisphere.}

Next, to validate the extent of DA cell loss two weeks after the administration of $1.85 \mathrm{mg} / \mathrm{ml}$ free-base dose of 6-OHDA, TH immunoreactivity (IR) was analysed. TH-IR was strongly decreased in ventral midbrain neurons in the lesioned hemisphere, suggesting DA neurons had been lost (Fig. 2A and B). SNc DA neurons were the most affected by the lesion with very few TH-positive neurons left. In contrast, TH-IR in the VTA was partially affected. The medial nuclei of the VTA remained intact corresponding to the interfascicular nucleus (IF), rostral linear nucleus (RLi) and the medial parts of the paranigral nucleus (PN) and parainterfascicular nucleus (PIF) while TH-positive neurons were decreased in the ventral and lateral nuclei of the VTA, the lateral part of the parabrachial pigmented nucleus (PBP) and ventro-lateral parts of the PN and PIF (Fig. 2A). The decrease in TH-positive fibers observed in the striatum directly matched the decrease of TH positive neurons in the midbrain. No TH-positive fibers were visible in the dorsal striatum (dStr), the primary target area of SNc DA neurons, on the lesioned side. Few TH positive fibers were detected in the ventral part of the nucleus accumbens shell (AcbSh) while strong TH immunepositive fibers were present in the medial part of the nucleus accumbens shell (mAcbSh) as well as the medial part of the olfactory tubercle (OT), these latter two the primary target areas of medial VTA DA neurons which were detected as preserved (Fig. 2B).

Still on the lesioned hemisphere, TH fibers were also preserved in structures known for receiving dopaminergic inputs either from other regions than the SNc/VTA or from noradrenergic (NA) areas (2729): the dorsal part of the bed nucleus of the stria terminalis (BNST), the central nucleus of the amygdala (CeA) and to a lesser extent the posterior basolateral nucleus of the amygdala (BLP) (Supplementary Fig. 1). In accordance with the preservation of these TH-positive fibers, TH-IR cell bodies were found in the paraventricular part of the posterior hypothalamus $(\mathrm{PH})$, the periaqueductal grey matter (PAG), the dorsal raphe nucleus (DRN) and the locus coeruleus (LC) (Supplementary Fig. 1).

The decrease of TH protein was confirmed by in situ hybridization experiments validating the presence of Th mRNA (Fig. 3). In addition to Th, also Vmat2 mRNA was used to confirm the degeneration of DA midbrain neurons. When comparing mice sacrificed two and three weeks post-surgery, the same amount of labeling was observed for both Th and Vmat2 mRNAs. Thus, based on these two markers for DA neurons, there is no difference in amount of cell death observed after only two weeks post-injection and 
when mice have been kept alive for three weeks after the injection (Fig. 3A-B for controls, 3A'-B' fot two weeks post-injection and $3 \mathrm{~A}^{\prime \prime}-\mathrm{B}$ " for three weeks post-injection).

\section{Calb1, Grp and Aldh1a1 mRNA detection levels are decreased in the right SNc and VTA two and three weeks after 6-OHDA lesioning.}

To better assess the extent of neuronal death in the midbrain, we next used three different molecular markers for subtypes of midbrain DA neurons: Calbindin 1 (Calb1), Gastrin-releasing peptide (Grp) and Aldehyde dehydrogenase 1 (Aldh1a1). Aldh1a1 has been described as primarily detected in DA neurons of the medial SNc and adjoining VTA, Calb1 is commonly used to define VTA DA neurons, while Grp has been reported in a modest subtype of VTA DA neurons (30-34).

First control mice were analyzed to verify the detection of SNc and VTA AD neuronal subypes as described in the literature. In control mice, Calb1 mRNA was confirmed to be present in all VTA subareas and to a much lesser extent in the SNc and lateral SNc (ISNc), as previously shown (32, 33) (Fig. 4A-A"). Grp mRNA was found in sparse neurons spatially confined to primarily the medial aspect of the VTA in accordance with literature (33). Grp mRNA was strongest in the PN, PIF and IF with weaker density in the PBP (Fig. 4B-B"). Aldh1a1 mRNA was detected in both the SNc and VTA with a higher density in the ventral SNc and medial aspect of the VTA; a lower density was detected in the dorsal PBP and lateral SNc (Fig. 4C-C"). Thus, in control mice, the patterns observed were same as described in the literature (32-34).

In contrast, all three molecular markers of VTA and SNc DA neuronal subtypes were strongly decreased in the 6-OHDA lesioned mice. The duration of the protocol (two or three weeks) did not visibly affect the severity of the lesion as detected by these markers. Instead, in both the two-week group and three-week group, the mRNA detection levels of the three markers were similar. Calb1 mRNA was nearly absent in the SNc and strongly decreased in the VTA on the lesioned hemisphere, particularly in the lateral parts of the PBP and ventral parts of the PN and PIF (Fig. 5D-D" and 5G-G"). Grp mRNA, restricted to the VTA in control mice, was decreased in the PBP and ventro-lateral parts of the PN and PIF (Fig. 5E-E" and 5H-H"). Aldh1a1 mRNA was decreased in both SNc and VTA in the lesioned hemisphere, however, few Aldh $1 \mathrm{a} 1+$ cells could be detected (Fig. 5F-F", 5I-I" and Supplementary Fig. 2). Taken together with the results of Th and Vmat2 above, these findings verify a substantial decrease of all markers analyzed for DA neurons in the SNc with prominent impact also on VTA DA neurons in the presented 6-OHDA lesion protocol.

\section{Discussion}

In this study, we present a complete protocol for the generation of the parkinsonian 6-OHDA mouse model which leads to successful deletion of midbrain DA neurons while preserving well-being and survival of the experimental animals. We believe that this protocol should prove beneficial to researchers wanting to study PD in mice, not least in the combination with viral-genetics methodology such as optogenetics, chemogenics and various fluorescent probes, for example. Here, the experimental procedure is presented using wild-type C57BL/6NTac mice to ascertain parameters and to provide a step-by-step protocol, but the improvements made should be applicable to additional mouse strains upon testing and validation. 
The 6-OHDA parkinsonian model is long-established to induce strong impairments in locomotion causing difficulties in feeding and hydrating, and leading to a low survival rate (35). We therefore tested different parameters to optimize the 6-OHDA model in mice. First, we implemented a daily post-operative care regime to prevent weight loss and dehydration. Second, the concentration of 6-OHDA was decreased compare to the usual doses used in the literature: the 6-OHDA concentration used in this study was 1.85 $\mathrm{mg} / \mathrm{ml}$ free-base while the usual concentration found in the literature varies between 2.5 to $3.6 \mathrm{mg} / \mathrm{ml}$ free-base (24-26). Third, we shortened the duration of the protocol to 14 days. This two-week protocol is, however, not always possible to apply if implementing behavioral experiments subsequent to the lesioning. Nevertheless, our results showed that 6-OHDA injected mice display strong parkinsonian features already two weeks post-lesion and with improved well-being, including feeding, should therefore be applicable in behavioral experiments. Further, for any terminal experiment, the two-week protocol should be sufficient for a complete study of down-stream events following DA cell death in this optimized mouse model of PD.

The 6-OHDA lesion caused a strong decrease of TH-IR in the DA cell bodies of the SNc and partially in the VTA. These results confirmed previous results showing that 6-OHDA injected in the MFB preferentially destroys DA neurons of the SNc compared to VTA DA neurons (36). The results were confirmed by in situ hybridization analyzing the presence of Th, Vmat2, Calb1, Grp and Aldh1a1 mRNA, known markers of dopaminergic neuronal populations and distinct DA subtypes in the VTA and SNc. Indeed, all mRNAs were decreased on the lesioned side in the SNc and ventro-lateral VTA, strongly suggesting that this was due to neuronal degeneration upon the 6-OHDA injection. In accordance with the loss of TH-IR in the ventral midbrain (SNc and VTA), TH-positive fibers were nearly absent from the dorsal and ventral striatum in the lesioned hemisphere, with the exception to the mAcbSh. However, the maintenance of TH fibers in this area is in full accordance with the preservation of DA neurons in the medial VTA, including the IF and medial PN, which project to this accumbal area. The strong decrease of TH observed with low concentration of 6-OHDA is in accordance with a previous study showing partial loss of TH-IR in mice injected with a $0.7 \mathrm{mg} / \mathrm{ml}$ 6-OHDA solution, analysed five weeks post-lesion (21). One study also showed a decrease of TH-IR in the striatum one week post-lesion but with a higher concentration $(35,37)$. Here, we combined a lower dose with short interval between injection and sacrifice to optimize the protocol in perspective of both well-being and survival, and hence, reliability of data.

Although the 6-OHDA model mimics many parkinsonian features, it also has limitations. Firstly, it does not induce the formation of Lewy bodies, an important hallmark of PD. Secondly, the 6-OHDA neurotoxin does not pass the blood brain barrier and has to be injected intracerebrally into the dorsal striatum, the MFB or the SNc to induce the degeneration of the nigrostriatal pathway and the associated PD symptoms. Thirdly, DA neurons of the VTA are also affected by the 6-OHDA but in a much lesser extent (38). This is per se not a limitation as it mimics the situation in human individuals, but an important factor to bear in mind when interpreting results using this model. Thus, while not representing the full repertoire of PD pathology observed in human individuals, the potent degeneration of SNc and VTA DA neurons in the 6-OHDA model makes it attractive for experimental studies centered around this critical 
aspect of PD. A mouse-based protocol with improved health status for the mice despite substantial DA cell death is therefore critical to achieve.

Limitation of the study: In general, studies that have implemented the 6-OHDA model in mice have rarely reported the survival rate of the animals, making it difficult for other researchers to assess the severity of the model in each setup. As a consequence, it has not been possible to make an exact comparison between studies and provise a precise estimate of the extent to which the presented protocol improves survival. Another limitation is the low amount of mice used in the current study which limits possibilities for statistical analysis. However, this limitation goes hand in hand with the animal welfare perspective of reducing the number of animals sacrificed for research pruposes. Further, by using a number of established markers for the DA neuron population, including more recently described markers for subtypes of DA neurons, the material has been carefully analyzed throughout each lesioned mouse brain.

In summary, the alleviation of post-operative symptoms and the high survival rate achieved using the current protocol could allow future studies to implement the 6-OHDA mouse model in combination with advanced experimental procedures aiming to identify and validate treatment methods for PD.

\section{Conclusions}

This study contributes to the improvement of experimental protocols of parkinsonian mouse models. By improving the 6-OHDA model in mice, future studies aiming to use this model can strive for, and achieve, using a lower number of mice while providing better living conditions, two main aspects of the $3 R$ principle in experimental animal welfare.

\section{Material And Methods}

\section{Animals and groups}

C57BL/6NTac (Taconic) male mice were used to generate and validate a 6-OHDA parkinsonian model. Mice were bred in-house and housed at the animal facility of Uppsala University. Mice had access to food and water ad libitum in standard humidity and temperature conditions and with a 12-hour dark/light cycle. All animal experimental procedures followed Swedish (Animal Welfare Act SFS 1998:56) and European Union Legislation (Convention ETS 123 and Directive 2010/63/EU).

Mice were divided in two groups according to the duration of the experimental protocol. One group of mice was sacrificed 14 days after $6-$ OHDA lesioning $(N=8)$ and a second group was sacrificed 21 days after 6-OHDA lesioning $(\mathrm{N}=7)$. Within each group, mice were divided in a 6-OHDA subgroup and a control subgroup (Two weeks post-lesion, $\mathrm{N}=4$ 6-OHDA mice, $\mathrm{N}=4$ control mice; Three weeks post-lesion $\mathrm{N}=46$ OHDA mice, $\mathrm{N}=3$ control mice). In addition to these 15 mice, several mice were injected with 6-OHDA for immunohistochemistry analysis $(\mathrm{N}=6)$.

\section{6-OHDA lesioning}


Mice were anesthetized with a mix of air and isoflurane $(4 \mathrm{~L} / \mathrm{min}$ isoflurane-air mix v/v) and maintained anesthetized with $0.5-2 \mathrm{~L} / \mathrm{min}$ of isoflurane. Mice were then placed in a stereotaxic apparatus and injected subcutaneously with an anti-inflammatory drug (Carprofen, $5 \mathrm{mg} / \mathrm{mL}$; Norocarp). After subcutaneous injection of a topical analgesic (Marcain, $1.5 \mathrm{mg} / \mathrm{kg}$; AstraZeneca), the skin was incised to expose the skull. A drill-hole was made above the MFB. Coordinates, on the right hemisphere: anteroposterior: $-1.20 \mathrm{~mm}$ from bregma and medio-lateral: $-1.10 \mathrm{~mm}$ from the sagittal vein. The 6-OHDA solution was freshly prepared on the day of injection by dissolving $2.2 \mathrm{mg}$ of 6-OHDA-hydrochloride in $0.9 \% \mathrm{NaCl} / 0.02 \%$ ascorbic acid solution (vehicle) and protected from the light. For control mice, $1 \mu \mathrm{L}$ of the vehicle was injected. $1 \mu \mathrm{L}$ of 6-OHDA solution $(1.85 \mathrm{mg} / \mathrm{mL})$ or vehicle was injected with a NanoFil syringe (World Precision Instruments, Sarasota, FL, USA) at $100 \mathrm{nl} / \mathrm{min}$, at $-4.75 \mathrm{~mm}$ from the brain's surface. After $10 \mathrm{~min}$, the needle was slowly removed and the skin stitched. Mice received another injection of Carprofen 20-24 hours after the surgery.

\section{Post-operative care}

The weight of the mice was monitored daily. Mice injected with 6-OHDA received subcutaneous injections of pre-heated saline $\left(35-37^{\circ} \mathrm{C}, 1 \mathrm{~mL}\right)$ every day until sacrifice to prevent dehydration and hypothermia. In addition, pellets dipped in a $15 \%$ sucrose solution were put in the home cages as well as an access to a petri dish filled with the same sucrose solution. Sunflower seeds and nutritionally fortified water gel were also used to prevent substantial weight loss and dehydration.

\section{Tissue preparation}

Two or three weeks after 6-OHDA injections, respectively, mice were sacrificed by cervical dislocation. Brains intended for histological analysis by in situ hybiridzation were frozen in -30 to $-35^{\circ} \mathrm{C}$ cold 2 methylbutane ( $\geq 99 \%$, Honeywell) and stored at $-80^{\circ} \mathrm{C}$ until sectioned with cryostat (Leica Microsystems, Germany ). For histological analysis by immunohistochemistry, whole-animal perfusion with $1 \mathrm{X}$ phosphate buffer saline (PBS) followed by ice-cold $4 \%$ formaldehyde (FA) was performed, whereupon brains were extracted, post-fixed in 4\% FA for 24 hours and cut with a vibratome (Leica Microsystems, Germany ).

\section{Immunohistochemistry}

$60 \mu \mathrm{m}$-thick coronal brain sections were washed and incubated for $15 \mathrm{~min}$ in a $1 \%$ hydrogen peroxidase solution diluted in 1X PBS 0.3\% TritonX-100 (0.3\% PBST) at room temperature (RT). After rinsing, sections were incubated for $90 \mathrm{~min}$ in a blocking solution containing $5 \%$ normal donkey serum (NDS, Millipore) and $0.3 \%$ PBST. Sections were then incubated with a primary antibody rabbit anti-TH (1:4000, AB152, Millipore) diluted in $0.3 \%$ PBST and $1 \%$ NDS overnight at $4^{\circ} \mathrm{C}$. The next day, sections were rinsed in $1 \mathrm{X}$ PBS and then incubated with a secondary antibody donkey anti-rabbit biotin-SP-conjugated (1:1000, AP182B, Millipore) diluted in 0.3\% PBST for 90 min at RT. After washes, an avidin/biotin-based peroxidase system (Vector ABC kit PK-6100, Vector Laboratories) was used to amplify the signal for 90 min followed by a pre-wash in Tris $0.1 \mathrm{M} \mathrm{pH7.4}$ for $10 \mathrm{~min}$. Sections were incubated in a DAB peroxidase solution (SK-4100, Vector Laboratories). Sections were then washed in Tris $0.1 \mathrm{M} \mathrm{pH7.4}$ for $10 \mathrm{~min}$ 
followed by two more washes in 1X PBS. The sections were mounted on glass slides, counterstained with cresyl violet, dehydrated with increasing concentrations of ethanol and mounted with a mounting medium (Leica CV Mount). Sections were scanned with NanoZoomer 2-0-HT.0 and visualized with NDP.view2 software (Hamamatsu).

\section{Fluorescent in situ hybridization (FISH)}

$16 \mu \mathrm{m}$-thin coronal sections were cut at the cryostat and mounted on glass slides for in situ hybridization. The protocol originally described in Viereckel et al, referred to as sdFISH (33), and briefly outlined below, was used to allow detection of two mRNAs in the same section. The sdFISH experiments were performed to detect the following mRNAs: Aldehyde dehydrogenase 1 (Aldh1a1), Calbindin1 (Calb1) and Gastrinreleasing peptide (Grp), all co-analyzed with Th mRNA. Probe design was described in Dumas and Wallén-Mackenzie and was produced by Oramacell (39). Sections were thawed and post-fixed in 4\% paraformaldehyde (4\% PFA) for 10 min at RT. After washes in 1X PBS, sections were incubated in a triethanolamine solution (TEA, pH 8) for 5 min followed by incubation in acetic anhydride solution (TEA). Riboprobes (DIG-labelled riboprobe, $50-75 \mathrm{ng} / 100 \mu \mathrm{l}$ and fluorescein-labelled riboprobe, $75-100 \mathrm{ng} / 100$ $\mu$ l) were denatured in hybridization buffer at $85^{\circ} \mathrm{C}$ for $10 \mathrm{~min}$ and applied on the sections for $16-18$ hours incubation at $65^{\circ} \mathrm{C}$ in a humidified chamber. After hybridization, sections were washed in $65^{\circ} \mathrm{C}$ saline sodium citrate buffer (SSC) baths ( 5 X SSC followed by $0.2 X$ SSC solutions) and a last wash with a $0.2 X$ SSC at RT. Sections were then washed in 1X maleic acid buffer containing TritonX-100 (MABT) to decrease the binding of non-specific probes. Fluorescein riboprobe was revealed by incubating the sections in a blocking solution containing a blocking reagent (BR, Roche), heat-inactivated FBS and $5 \mathrm{X}$ $M A B$. Sections were then incubated with an anti-fluorescein horseradish peroxidase conjugated (POD) antibody (Roche) for 1 hour. An amplification step was performed by incubating the slices in TSA-biotin amplification buffer (Perkin Elmer) for $15 \mathrm{~min}$. Fluorescein-TSA-biotin complex was revealed by adding Neutravidin-Oregon Green (Invitrogen) for $15 \mathrm{~min}$. After rinsing the sections in TritonX-100 PBS (PBST), hydrogen peroxidases were inhibited by incubation in $0.1 \mathrm{M}$ glycine $(\mathrm{pH} 2.1)$ and $3 \%$ hydrogen peroxide $\left(\mathrm{H}_{2} \mathrm{O}_{2}\right)$. A similar procedure was followed to subsequently reveal the DIG riboprobe with an anti-DIG-POD antibody diluted in BR solution and TSA buffer plus Cy3 (Perkin Elmer). Sections were incubated for 10 min in 1/50000 DAPI solution and mounted with Fluoromount (Southern Biotech). Slices were scanned with NanoZoomer 2-0-HT.0 scanner using the NDP.scan 3.3 software (Hamamatsu).

Mice injected with 6-OHDA received subcutaneous injections of pre-heated saline $\left(30-35^{\circ} \mathrm{C}, 1 \mathrm{~mL}\right)$ every day until sacrifice to prevent dehydration and hypothermia. In addition, pellets dipped in a $15 \%$ sucrose solution were put in the home cages as well as an access to a petri dish filled with the same sucrose solution. Sunflower seeds and nutritionally fortified water gel were also used to prevent substantial weight loss and dehydration.

\section{Declarations}

\section{Ethics approval and consent to participate}


The animal study was reviewed and approved by Uppsala Ethical Committee for Laboratory Animal Research (Uppsala djurförsöksetiska nämnd Uppsala Tingsrätt Box 111375141 Uppsala).

\section{Consent for publication}

Not applicable

\section{Availability of data and materials}

The datasets supporting the conclusions of this article are included within the article and its additional files. Correspondence and request for additionnal data or material should be addressed to the corresponding author on reasonable request.

\section{Competing interests}

All authors declare no conflict of interests

\section{Funding}

This work was funded by Uppsala University and by grants to A.W.M from the Swedish Research Council (SMRC 2017-02039), the Swedish Brain Foundation (Hjärnfonden), Parkinsonfonden, and the Research Foundations of Bertil Hållsten, Zoologiska stiftelsen and Åhlén.

\section{Authors' contributions}

AG: Investigation, formal analysis, methodology, writing - original draft, review and editing; BV: Investigation, formal analysis;. ÅM: Conceptualization, formal analysis, funding acquisition, project administration, supervision, writing - review and editing.

\section{Acknowledgments}

We thank Dr Sylvie Dumas (Oramacell) for providing primers and probes for the in situ hybridization experiments.

\section{References}

1. Tysnes O-B, Storstein A. Epidemiology of Parkinson's disease. J Neural Transm. 2017 Aug;124(8):901-5.

2. Obeso JA, Stamelou M, Goetz CG, Poewe W, Lang AE, Weintraub D, et al. Past, present, and future of Parkinson's disease: A special essay on the 200th Anniversary of the Shaking Palsy: The Shaking Palsy: Past, Present and Future. Mov Disord. 2017 Sep;32(9):1264-310.

3. Khan AU, Akram M, Daniyal M, Zainab R. Awareness and current knowledge of Parkinson's disease: a neurodegenerative disorder. International Journal of Neuroscience. 2019 Jan 2;129(1):55-93. 
4. Spillantini MG, Schmidt ML, Lee VM-Y, Trojanowski JQ, Jakes R, Goedert M. a-Synuclein in Lewy bodies. Nature. 1997 Aug;388(6645):839-40.

5. Braak H, Tredici KD, Rüb U, de Vos RAl, Jansen Steur ENH, Braak E. Staging of brain pathology related to sporadic Parkinson's disease. Neurobiology of Aging. 2003 Mar;24(2):197-211.

6. Barker RA, Williams-Gray CH. Review: The spectrum of clinical features seen with alpha synuclein pathology. Neuropathol Appl Neurobiol. 2016 Feb;42(1):6-19.

7. Kordower JH, Olanow CW, Dodiya HB, Chu Y, Beach TG, Adler CH, et al. Disease duration and the integrity of the nigrostriatal system in Parkinson's disease. Brain. 2013 Aug;136(8):2419-31.

8. Grealish S, Diguet E, Kirkeby A, Mattsson B, Heuer A, Bramoulle Y, et al. Human ESC-Derived Dopamine Neurons Show Similar Preclinical Efficacy and Potency to Fetal Neurons when Grafted in a Rat Model of Parkinson's Disease. Cell Stem Cell. 2014 Nov;15(5):653-65.

9. Cyranoski D. 'Reprogrammed' stem cells implanted into patient with Parkinson's disease. Nature. 2018 Nov 14; d41586-018-07407-9.

10. Parmar M, Grealish S, Henchcliffe C. The future of stem cell therapies for Parkinson disease. Nat Rev Neurosci. 2020 Feb;21(2):103-15.

11. Alexander GE, Crutcher MD, DeLong MR. Basal ganglia-thalamocortical circuits: parallel substrates for motor, oculomotor, 'prefrontal' and 'limbic' functions. Prog Brain Res. 1990;85:119-46.

12. Benabid AL, Pollak P, Gross C, Hoffmann D, Benazzouz A, Gao DM, et al. Acute and Long-Term Effects of Subthalamic Nucleus Stimulation in Parkinson's Disease. Stereotactic and Functional Neurosurgery. 1994;62(1-4):76-84.

13. Heywood P, Gill S. Bilateral dorsolateral subthalamotomy for advanced Parkinson's disease. The Lancet. 1997 Oct;350(9086):1224.

14. Kim H-J, Jeon BS, Paek SH. Nonmotor Symptoms and Subthalamic Deep Brain Stimulation in Parkinson's Disease. JMD. 2015 May 31;8(2):83-91.

15. Petry-Schmelzer JN, Krause M, Dembek TA, Horn A, Evans J, Ashkan K, et al. Non-motor outcomes depend on location of neurostimulation in Parkinson's disease. Brain. 2019 Nov 1;142(11):3592-604.

16. Accolla EA, Pollo C. Mood Effects After Deep Brain Stimulation for Parkinson's Disease: An Update. Front Neurol. 2019;10:617.

17. Lorenc-Koci E, Wolfarth S, Ossowska K. Haloperidol-increased muscle tone in rats as a model of parkinsonian rigidity. Exp Brain Res [Internet]. 1996 May [cited 2021 Apr 12];109(2). Available from: 
18. Duty S, Jenner P. Animal models of Parkinson's disease: a source of novel treatments and clues to the cause of the disease: Animal models of Parkinson's disease. British Journal of Pharmacology. 2011 Oct;164(4):1357-91.

19. Triarhou LC. Introduction. Dopamine and Parkinson's disease. Adv Exp Med Biol. 2002;517:114.

20. Cenci MA, Björklund A. Animal models for preclinical Parkinson's research: An update and critical appraisal. In: Progress in Brain Research [Internet]. Elsevier; 2020 [cited 2021 May 4]. p. 27-59. Available from: https://linkinghub.elsevier.com/retrieve/pii/S0079612320300200

21. Boix J, Padel T, Paul G. A partial lesion model of Parkinson's disease in mice - Characterization of a 6-OHDA-induced medial forebrain bundle lesion. Behavioural Brain Research. 2015 May;284:196206.

22. Park SE, Song K-I, Kim H, Chung S, Youn I. Graded 6-OHDA-induced dopamine depletion in the nigrostriatal pathway evokes progressive pathological neuronal activities in the subthalamic nucleus of a hemi-parkinsonian mouse. Behav Brain Res. 2018 May 15;344:42-7.

23. Glinka Y, Gassen M, Youdim MBH. Mechanism of 6-hydroxydopamine neurotoxicity. In: Riederer P, Calne DB, Horowski R, Mizuno Y, Poewe W, Youdim MBH, editors. Advances in Research on Neurodegeneration [Internet]. Vienna: Springer Vienna; 1997 [cited 2021 Mar 29]. p. 55-66. (Journal of Neural Transmission. Supplementa; vol. 50). Available from: http://link.springer.com/10.1007/978-37091-6842-4_7

24. Francardo V, Recchia A, Popovic N, Andersson D, Nissbrandt H, Cenci MA. Impact of the lesion procedure on the profiles of motor impairment and molecular responsiveness to L-DOPA in the 6hydroxydopamine mouse model of Parkinson's disease. Neurobiology of Disease. 2011 Jun;42(3):32740.

25. Sanders $\mathrm{TH}$, Jaeger D. Optogenetic stimulation of cortico-subthalamic projections is sufficient to ameliorate bradykinesia in 6-ohda lesioned mice. Neurobiology of Disease. 2016 Nov;95:225-37.

26. Bagga V, Dunnett SB, Fricker RA. The 6-OHDA mouse model of Parkinson's disease - Terminal striatal lesions provide a superior measure of neuronal loss and replacement than median forebrain bundle lesions. Behavioural Brain Research. 2015 Jul;288:107-17.

27. Canteras NS, Simerly RB, Swanson LW. Connections of the posterior nucleus of the amygdala. J Comp Neurol. 1992 Oct 8;324(2):143-79.

28. Hasue RH, Shammah-Lagnado SJ. Origin of the dopaminergic innervation of the central extended amygdala and accumbens shell: A combined retrograde tracing and immunohistochemical 
study in the rat. J Comp Neurol. 2002 Dec 2;454(1):15-33.

29. Matthews GA, Nieh EH, Vander Weele CM, Halbert SA, Pradhan RV, Yosafat AS, et al. Dorsal Raphe Dopamine Neurons Represent the Experience of Social Isolation. Cell. 2016 Feb;164(4):617-31.

30. Chung CY, Seo H, Sonntag KC, Brooks A, Lin L, Isacson O. Cell type-specific gene expression of midbrain dopaminergic neurons reveals molecules involved in their vulnerability and protection. Human Molecular Genetics. 2005 Jul 1;14(13):1709-25.

31. Greene JG, Dingledine R, Greenamyre JT. Gene expression profiling of rat midbrain dopamine neurons: implications for selective vulnerability in parkinsonism. Neurobiology of Disease. 2005 Feb;18(1):19-31.

32. Poulin J-F, Zou J, Drouin-Ouellet J, Kim K-YA, Cicchetti F, Awatramani RB. Defining Midbrain Dopaminergic Neuron Diversity by Single-Cell Gene Expression Profiling. Cell Reports. 2014 Nov;9(3):930-43.

33. Viereckel T, Dumas S, Smith-Anttila CJA, Vlcek B, Bimpisidis Z, Lagerström MC, et al. Midbrain Gene Screening Identifies a New Mesoaccumbal Glutamatergic Pathway and a Marker for Dopamine Cells Neuroprotected in Parkinson's Disease. Sci Rep. 2016 Dec;6(1):35203.

34. Poulin J-F, Caronia G, Hofer C, Cui Q, Helm B, Ramakrishnan C, et al. Mapping projections of molecularly defined dopamine neuron subtypes using intersectional genetic approaches. Nat Neurosci. 2018 Sep;21(9):1260-71.

35. Thiele SL, Warre R, Nash JE. Development of a Unilaterally-lesioned 6-OHDA Mouse Model of Parkinson's Disease. JoVE. 2012 Feb 14;(60):3234.

36. Tan Y, Williams EA, Lancia AJ, Zahm DS. On the altered expression of tyrosine hydroxylase and calbindin-D 28kD immunoreactivities and viability of neurons in the ventral tegmental area of Tsai following injections of 6-hydroxydopamine in the medial forebrain bundle in the rat. Brain Research. 2000 Jun;869(1-2):56-68.

37. Rentsch P, Stayte S, Morris GP, Vissel B. Time dependent degeneration of the nigrostriatal tract in mice with 6-OHDA lesioned medial forebrain bundle and the effect of activin A on I-Dopa induced dyskinesia. BMC Neurosci. 2019 Dec;20(1):5.

38. Heuer A, Smith GA, Lelos MJ, Lane EL, Dunnett SB. Unilateral nigrostriatal 6-hydroxydopamine lesions in mice I: Motor impairments identify extent of dopamine depletion at three different lesion sites. Behavioural Brain Research. 2012 Mar;228(1):30-43.

39. Dumas S, Wallén-Mackenzie Å. Developmental Co-expression of Vglut2 and Nurr1 in a Mes-DiEncephalic Continuum Preceeds Dopamine and Glutamate Neuron Specification. Front Cell Dev Biol. 2019 Nov 28;7:307. 


\section{Tables}

Due to technical limitations, table 1 is only available as a download in the Supplemental Files section.

\section{Figures}
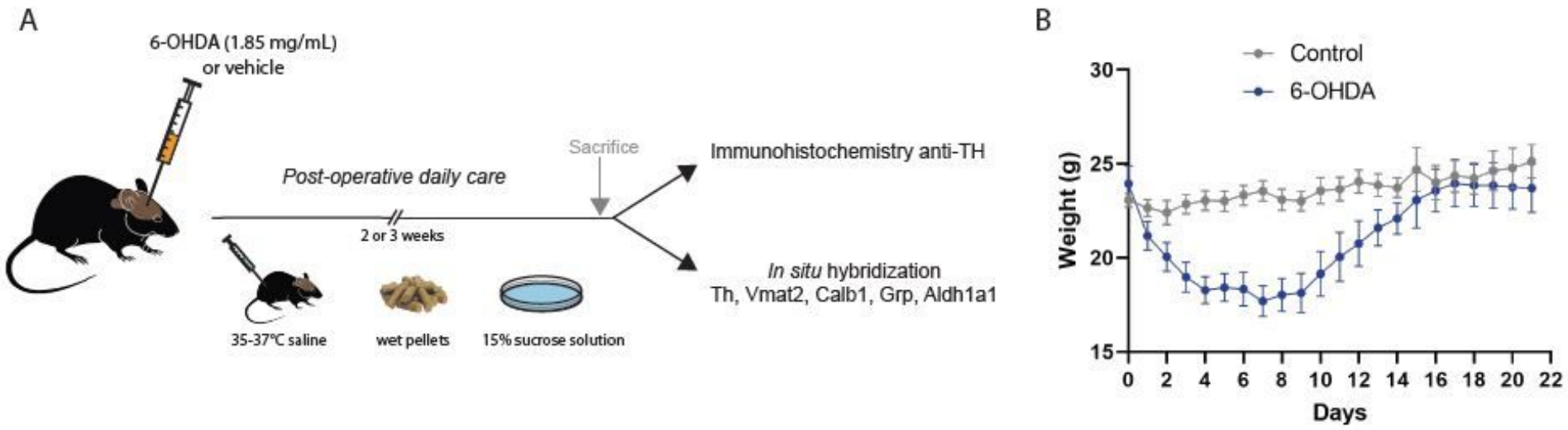

Figure 1

Experimental protocol to induce parkinsonian state in wild-type mice. (A) Post-operative care included daily subcutaneous injection of warm saline, access to pellets dipped in a $15 \%$ sucrose solution and access to a petri dish filled with $15 \%$ sucrose solution placed inside the home cage. Mice were sacrificed two or three weeks after 6-OHDA injection in the medial forebrain bundle (MFB). Immunohistochemistry and in situ hybridization where performed to detect dopaminergic markers. (B) Weight evolution of the 6OHDA lesioned mice and controls ( $\mathrm{N}=15$ from day 0 to $14 ; \mathrm{N}=8$ from day 15 to 21 ).

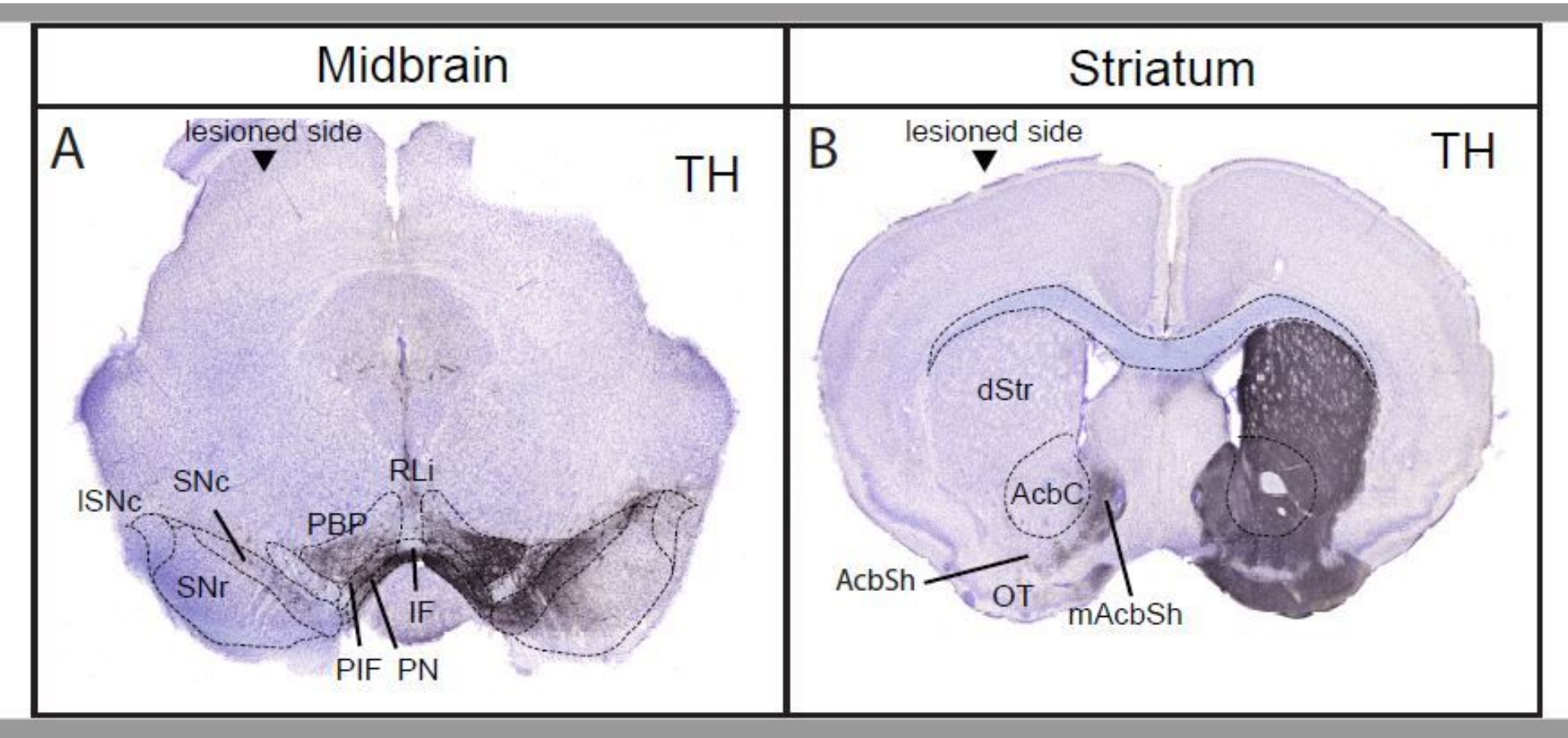

Figure 2 
Strong decrease of TH immunoreactivity in the nigrostriatal pathway. Representative immunohistochemistry anti-TH on coronal slices at the midbrain (A) and striatum (B) levels in a 6-OHDA lesioned mouse. 6-OHDA: 6-hydroxydopamine; ISNc: lateral substantia nigra pars compacta; SNc: substantia nigra pars compacta; SNr: substantia nigra pars reticulata; PBP: parabrachial pigmented nucleus; PIF: parainterfascicular nucleus; PN: paranigral nucleus; IF: interfascicular nucleus; RLi: rostral linear nucleus; dStr: dorsal striatum; AcbC: nucleus accumbens core; mAcbSh: medial part of the nucleus accumbens shell; OT: olfactory tubercle.


\section{Figure 3}

Strong decrease of Th and Vmat2 mRNAs in the ventral midbrain in the lesioned hemisphere. (A and $B$ ) mRNA expression of Th (A) and Vmat2 (B) in the SNc and VTA in control mice. ( $A^{\prime}$ and $B^{\prime}$ ) mRNA expression of Th ( $\left.A^{\prime}\right)$ and Vmat2 ( $\left.B^{\prime}\right)$ in the SNc and VTA in 6-OHDA injected mice, 2 weeks post-lesioned. ( $A^{\prime \prime}$ and $\left.B^{\prime \prime}\right)$ mRNA expression of Th $\left(A^{\prime \prime}\right)$ and Vmat2 $\left(B^{\prime \prime}\right)$ in the SNc and VTA in 6-OHDA injected mice, 3 weeks post-lesioned. Scale bar: $500 \mu \mathrm{m}$. 


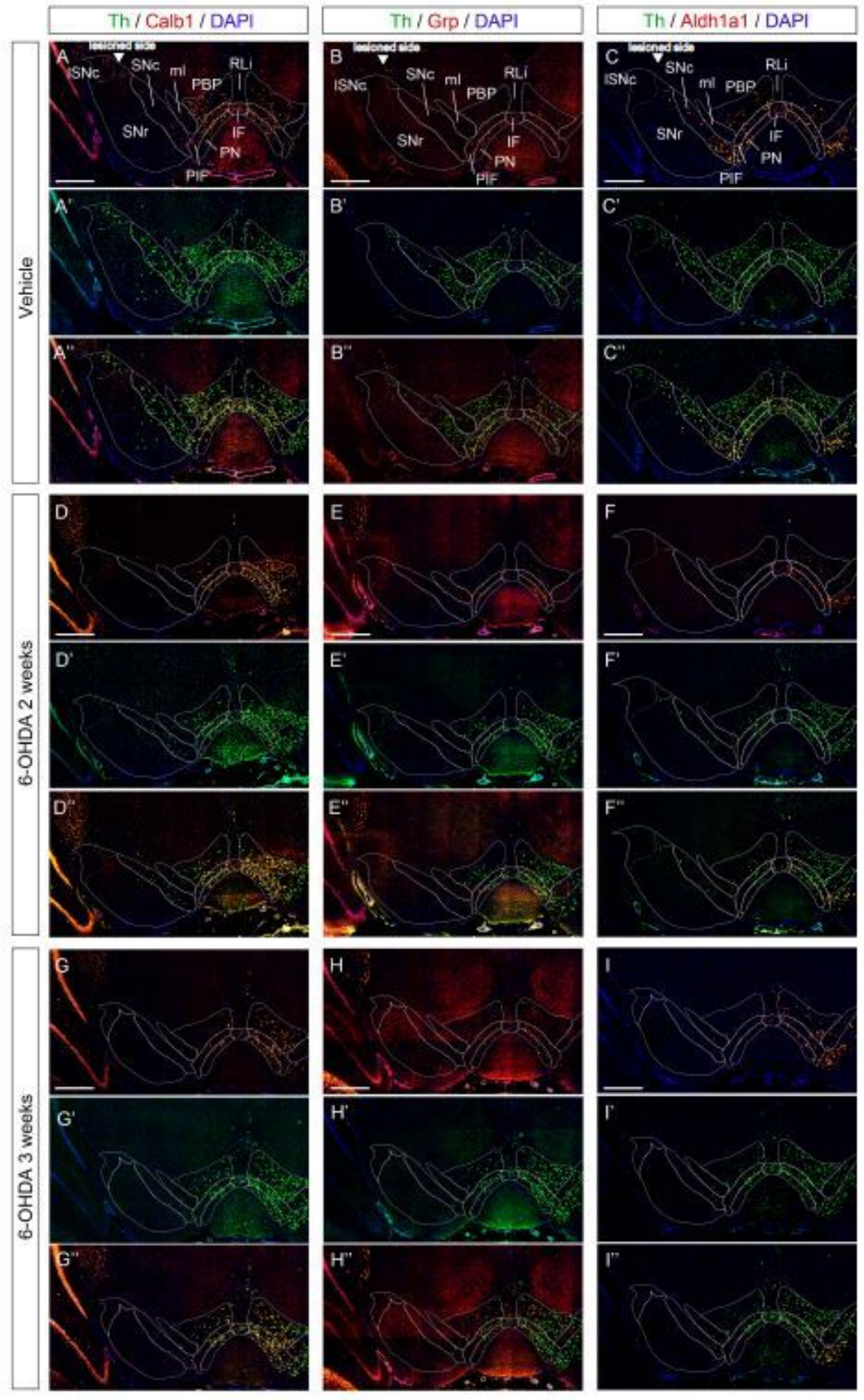

\section{Figure 4}

Detection of mRNA of dopaminergic midbrain molecular markers in 6-OHDA lesioned mice and control mice. (A-A") Calb1 (red) and Th (green) mRNAs in the ventral midbrain, in control mice, (D-D") 2 weeks after 6-OHDA injection and (G-G") 3 weeks after 6-OHDA injection. (B-B") Grp (red) and Th (green) mRNAs in the ventral midbrain, in control mice, (E-E") 2 weeks after 6-OHDA injection and $\left(\mathrm{H}-\mathrm{H}^{\prime \prime}\right) 3$ weeks after 6OHDA injection. (C-C") Aldh1a1 (red) and Th (green) mRNAs in the ventral midbrain, in control mice, (F-F") 
2 weeks post 6-OHDA injection and (II") 3 weeks post 6-OHDA injection. ISNc: lateral substantia nigra pars compacta; SNc: substantia nigra pars compacta; SNr: substantia nigra pars reticulata; PBP: parabrachial pigmented nucleus; PIF: parainterfascicular nucleus; PN: paranigral nucleus; IF: interfascicular nucleus; RLi: rostral linear nucleus; ml: medial lemniscus. Scale bar: $500 \mu \mathrm{m}$.

\section{Supplementary Files}

This is a list of supplementary files associated with this preprint. Click to download.

- Additionalfile1.docx

- Supplementaryfigure1.jpg

- Supplementaryfigure2.pdf

- Supplementarytable1.tif

- Table1.pdf 